\title{
Proteomics Exploration of Chronic Lymphocytic Leukemia
}

\section{ISSN: 2637-773X}

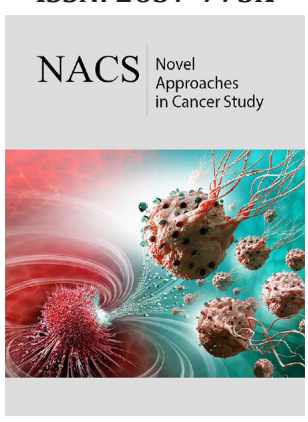

*Corresponding author: Tiara Griffen, Department of Microbiology, Biochemistry, and Immunology, Morehouse School of Medicine, Atlanta, GA 30310, USA

Submission: 㓪 July 11, 2019

Published: 眥July 16, 2019

Volume 3 - Issue 1

How to cite this article: Tiara G, Steven K. Proteomics Exploration of Chronic Lymphocytic Leukemia. Nov Appro in Can Study. 3(1). NACS.000553.2019. DOI: 10.31031/NACS.2019.03.000553

Copyright@ Tiara Griffen, This article is distributed under the terms of the Creative Commons Attribution 4.0 International License, which permits unrestricted use and redistribution provided that the original author and source are credited.

\author{
Tiara L Griffen ${ }^{1 *}$ and Steven M Kornblau ${ }^{2}$ \\ ${ }^{1}$ Department of Microbiology, Biochemistry, and Immunology, Morehouse School of Medicine, \\ Atlanta, GA 30310, USA \\ ${ }^{2}$ Department of Leukemia, The University of Texas MD Anderson Cancer Center, Houston, TX, \\ USA 77030
}

\begin{abstract}
Chronic Lymphocytic Leukemia (CLL) is an adult heme malignancy characterized by the presence of mature-appearing CD5+ B cells in the blood, bone marrow, and secondary lymphoid organs. CLL is heterogeneous in its progression and clinical outcomes. Factors that contribute to the heterogeneity include the immunoglobulin heavy chain (IGHV) status and chromosomal aberrations. CLL's heterogeneous behavior has made it difficult to understand its biology and find a cure. Systems biology approaches are commonly used to establish biomarkers for cancer. Because proteins are targets of FDA approved therapies, proteomics approaches have been used to decipher the c mplex behavior of CLL pathogenesis and identify actionable targets. In this minireview, we highlight factors that contribute to CLL, new insights discovered from proteomics studies, and future directions.
\end{abstract}

\section{Introduction}

Chronic Lymphocytic Leukemia (CLL) is an adult hematological malignancy characterized by the presence of mature-appearing CD5+ B cells in the blood, bone marrow, and secondary lymphoid organs [1]. In the United States, there will be an estimate of 20,720 new cases and 3,930 deaths according to the American Cancer Society statistics. Symptoms include swollen lymph nodes, frequent infections, and fatigue which negatively impacts the quality of life of people affected [1]. CLL is heterogeneous in its progression and clinical outcomes. Factors that contribute to the heterogeneity include the immunoglobulin heavy chain (IGHV) status and chromosomal aberrations [2,3]. The IGHV mutation status, stratified as $>$ or $<2 \%$ of cell with the mutation[4], can be used to define two subtypes of CLL: Unmutated(U-CLL) and Mutated CLL(M-CLL) with $40 \%$ and $60 \%$ of patients respectively. U-CLL is characterized by the presence of CLL cells that have less than two percent of their IGHV mutated, whereas M-CLL cells have more than two percent mutated [4]. U-CLL is the more aggressive phenotype [2] having increased responsiveness to antigens that bind the B cell receptor (BCR) versus M-CLL cells [5]. M-CLL is the more indolent phenotype. Increased BCR signaling results in increased cell survival and proliferation [5].

Mutations that influence CLL's progression include the $17 \mathrm{p}$ deletion(p53), trisomy 12(NOTCH1), 13q deletion(mir15), deletion 11q(ATM), and SF3B1[6]. These mutations result in the loss of p53, increased NOTCH1 expression, increased BCL-2 expression, and loss of ATM. NOTCH1 is mutated in $10-15 \%$ of patients diagnosed with CLL. NOTCH1 has a role in the development of different types of blood cells. NOTCH1 amplification results in faster progression, a less favorable outcome, and have increased risk of Richter's transformation, the process of CLL cells transforming into diffuse large B cell lymphoma [7]. SF3B1 is a splicing factor subunit and has a role in alternative mRNA splicing and formation of certain proteins in CLL and other hematological malignancies [8]. The SF3B1 mutation is commonly found in patients with U-CLL and high expression of CD38 and ZAP-70 and is associated with shorter treatment free survival [9]. 10-15\% of patients have a mutation in this gene resulting in dysfunctional protein processing [8]. This mutation is associated with fludarabine therapy resistance. p53 is an important part in the cell cycle checkpoint. It is part of the DNA damage response. This mutation is associated with increased growth and resistance to chemotherapy [10].

Factors that are associated with high-risk CLL progression are the high expression of CD38, Beta2-microglobulin, and ZAP-70. CD38 is a cell surface protein on immune and CLL cells that 
express CD38 are more responsive to BCR stimulation and exhibit enhanced migration and increased proliferation and chemotaxis via ZAP-70 and ERK 1/2 signaling [11]. Beta 2-microglobulin(b2-m) is a protein that is a component of the MHC class I complex. High b2-m is associated with advanced stage and high lymphocyte counts in CLL [12]. ZAP-70 is a protein expressed near the surface of $\mathrm{T}$ cells and has an important role in $\mathrm{T}$ cell signaling. Abnormal ZAP-70 expression is associated with increased BCR signaling and migration towards tumor microenvironment in CLL and shorter survival $[13,14]$. Patients with high expression of ZAP-70, CD38, and beta 2-microglobulin have a poorer prognosis.

Because CLL has a variety offactors that influence its progression and heterogeneity, it is important to identify how these factors alter B cell behavior on a systems level. Systems level approaches include genomics, transcriptomic, epigenetics, metabolomics, and proteomics. Although there is much insight provided by the various omics methods, proteins are the final product and the targets of many FDA approved therapiesand therefore may be more relevant to study. Furthermore, previous studies have reported low correlations between protein and gene expression. Hence, we should focus on exploring the post-translationally modified proteins to define and understand cancer biology. High throughput proteomics methods consist of multiplex immunoassays, phospho-specific flow cytometry, reverse phase protein arrays, quantitative immunohistochemistry, and mass spectrometry [15]. Phosphorylation specific flow cytometry is a high throughput method that quantifies phosphorylation levels of many cellular proteins [15]. Quantitative immunohistochemistry is a method that uses fluorescent labels to determine tumor and stroma localization, of proteins of interest [15]. Mass Spectrometry quantifies low and large abundance phospho-proteins. Multiplex immunoassays and Reverse Phase protein arrays allow the detection of proteins, with antibodies, from hundreds of analytes simultaneously and uses a small amount of sample [15]. The purpose of this review is to discuss proteomics methodologies used in CLL research and the insights that they have brought.

\section{Outcomes of CLL Proteomics Research: Deciphering CLL Metabolism and Tumor Microenvironment Signaling}

Deregulated cellular metabolism is an established hallmark of cancer [16]. Cancer cells derived from different tissues, have heterogeneous metabolic signaling. Proteomics methods have been ideal for quantifying metabolic signaling molecules to define cancer specific signatures. Mayer et al. Performed a study using mass spectrometry on 16 CLL and healthy donor samples from younger and older patients [17]. This study provided insight in the differences in the expression of proteins involved in the hallmarks of cancer. They observed that proteins involved in the inflammatory response, mitochondrial metabolism, DNA damage response, and mitochondrial stress and aging were deregulated in elderly patients versus the young patients. When assessing expression of proteins involved in the hallmarks of cancer, CLL cells had increased expression of proteins involved in "genome instability and mutation", "avoiding immune destruction", "resisting cell death", and "deregulation of cellular energies". [17]

Thurgood et al. performed a study using a mass spectrometry analysis of PBMC(n=3) and healthy(n=6) or CLL(n=12) lymph node samples $(\mathrm{n}=12)$ and compared the results to healthy controls. [18] 12 and 3 CLL and healthy PBMC samples were included. 14 CLL and 6 healthy lymph nodes were examined. They observed that CLL cells had upregulation of proteins involved in ROS formation, the anti-oxidant response, and an auto-immune response that initiates hemolytic anemia compared to healthy controls. Differential expression analysis between the U-CLL and M-CLL samples revealed in 60 downregulated and 133 upregulated proteins. The upregulated proteins were involved in BCR stimulation and ROS production, consistent with previous report of increased BCR signaling in U-CLL. Pathways associated with the 189 differentially expressed proteins include migration, stress, PI3K, and glycolytic/ fatty acid signaling. Differential expression analysis of CLL and healthy lymph nodes revealed increased expression of CD99, STAT3, and anti-oxidant proteins. The authors also observed that there was a shift towards increased fatty acid and cholesterol synthesis in CLL cells.

CLL progression and survival is highly dependent on interactions within the tumor immune microenvironment [19]. The microenvironment consists of $\mathrm{T}$, stromal, endothelial, and nurse-like cells. These cells provide essential signaling molecules such as chemokines, WNT, and interleukins for cells survival and proliferation. CLL cell dependency on the microenvironment has made it difficult to culture cells once they are removed from patients. The roles that each of these cell types play in CLL progression have not been defined. To identify the role of stromal cells in CLL progression, Vangapandu et al. [20] used RPPA on peripheral blood CLL cells that were co-cultured with stromal cells. CLL cells that were cultured with stromal cells have significant [20] increase in calveolin-1, STAT3, NFkB, PDGFR, and cyclin B1 versus CLL cells alone. Increased expression of NFkB and STAT3 are associated with aggressive CLL progression. The secretion of PDGF was previously shown to mediate the expansion of mesenchymal stem cells in the tumor microenvironment. This study established that the stromal cells aid in the malignant growth of CLL cells and increases interactions with cells in the tumor microenvironment.

A deregulated immune response is a key symptom of CLL. The origin of this deregulated immune response is currently unknown. Some hypothesize that CLL B cell expansion originated in response to a bacterial or viral infection or an auto-antigen. Griggio et al. identified cancer associated antigens in CLL that are recognized by patient antibodies.[21] They obtained these results from a serological proteome analysis (SERPA) of sera obtained from 35 patients who were untreated.

SERPA is a combination method that consists of gel electrophoresis, western blotting, and mass spectrometry. Proteins recognized by antibodies, in patient sera, include EN01, G3P 
dehydrogenase, pyruvate dehydrogenase E1 subunit, and an actin binding protein. Out of all the proteins recognized, ENO1 was the only protein that was significantly recognized. ENO1 was also found to be overexpressed in lymph node tissues, expressed intracellularly in peripheral blood derived CLL cells, and present on the surface of apoptotic B cells. The authors also noted that there was increased immunoreactivity in samples that had the high expression of ZAP70 and CD38. CLL cell recognition of these antigen promotes increased BCR signaling, hence increasing proliferation and survival, and establishing a chronic disease state.

\section{Conclusion}

Proteomic studies provide information on what proteins are actually being synthesized and post-translationally modified in normal and disease states. Examination of mutations in DNA are resourceful, but not if they do not have a consequence on the final protein product. Transcriptome information is also really good, but not all genes are translated into proteins. Proteins are the primary targets for cancer therapy. Proteomics studies have revealed new findings on the protein expression of CLL cells in the blood and lymph nodes along with young and old patients. These analyses have also brought new knowledge on the interactions between CLL cells and its tumor microenvironment and possible antigens that drive the chronic disease state. Although there have been many insights from past CLL proteomics studies, there is much left to do to define its pathogenic biology to find a cure. The aforementioned CLL protein studies were performed with mass spectrometry and small sample sizes, which is inefficient for proving a hypothesis. Also, the attributes examined in these studies did not reflect differences in patient protein expression reported based on their mutation, progression, and therapy status. Mutation, therapy, and progression status are prognostic factors that should be examined to establish signatures that can be used in a clinical setting. Including these factors will create a study that is truly representative of the heterogeneous patient population.

Recurrent mutation, metabolic, and gene signatures that have been identified that affect response,resistance, and survival status, but not protein signatures. It is important to identify protein signatures, since proteins are the actionable targets for FDA approved drugs. Resistance in CLL is typically attributed tomutations in BTK, PLCg, and BCL-2 proteins. Proteomics has the advantage identifying protein expression patterns associated with patients who have resistance status. For instance, Walliser et al. used proteomics to identify proteins associated with ibrutinibresistant patients with mutations in PLCg. They discovered that PLCg is sensitive to RAC2 expression. Targeting RAC2 could be a novel therapy target for patients with the mutated PLCg, making their CLL cells more sensitive to ibrutinib therapy[22]. Information from proteomics studies has the potential to be used to identify novel therapy targets to improve treatment options. To validate previous findings and to gather more insight, a large patient cohort, that is truly representative of CLL heterogeneity, should be analyzed. Future CLL proteomics studies should also include more analyses of post translational modifications, extensive clinical phenotype associations, analysis of cells from the tumor immune microenvironment, and observe changes in protein expression over time.

\section{References}

1. Kipps TJ, Stevenson FK, Wu CJ, Croce CM, Packham G, et al. (2017) Chronic lymphocytic leukaemia. Nat Rev Dis Primers 3: 16096.

2. Hamblin TJ, Davis Z, Gardiner A, Oscier DG, Stevenson FK (1999) Unmutated $\operatorname{Ig} \mathrm{V}(\mathrm{H})$ genes are associated with a more aggressive form of chronic lymphocytic leukemia. Blood 94(6): 1848-1854.

3. Damle RN, Wasil T, Fais F, Ghiotto F, Valetto A, et al. (1999) Ig V gene mutation status and CD38 expression as novel prognostic indicators in chronic lymphocytic leukemia. Blood 94(6): 1840-1847.

4. Fais F, Ghiotto F, Hashimoto S, Sellars B, Valetto A, et al. (1998) Chronic lymphocytic leukemia B cells express restricted sets of mutated and unmutated antigen receptors. J Clin Invest. 102(8): 1515-1525.

5. Burger JA Chiorazzi N (2013) B cell receptor signaling in chronic lymphocytic leukemia. Trends Immunol 34(12): 592-601.

6. Amin NA, Malek SN (2016) Gene mutations in chronic lymphocytic leukemia. Semin Oncol 43(2): 215-221.

7. Fabbri G, Khiabanian H, Holmes AB, Wang J, Messina M, et al. (2013) Genetic lesions associated with chronic lymphocytic leukemia transformation to Richter syndrome. J Exp Med 210(11): 2273-2288.

8. Obeng EA, Chappell RJ, Seiler M, Chen MC, Campagna, et al. (2016) Physiologic expression of Sf3b1(K700E) causes impaired erythropoiesis, aberrant splicing, and sensitivity to therapeutic spliceosome modulation. Cancer Cell 30(3): 404-417.

9. Miao Y, Zou YX, Gu DL1, Zhu HC, Zhu HY, et al. (2019) SF3B1 mutation predicts unfavorable treatment-free survival in Chinese chronic lymphocytic leukemia patients. Ann Transl Med 7(8): 176.

10. Rossi D, Khiabanian H, Spina V, Ciardullo C, Bruscaggin A, et al. (2014) Clinical impact of small TP53 mutated subclones in chronic lymphocytic leukemia. Blood 123(14): 2139-2147.

11. Malavasi F, Deaglio S, Damle R, Cutrona G, Ferrarini M, et al. (2011) CD38 and chronic lymphocytic leukemia: a decade later. Blood 118(13): 34703478 .

12. Berrebi A Bassous L, Haran M, Shtalrid M, Shvidel L (2010) The significance of elevated beta 2-microglobulin (b2-m) in chronic lymphocytic leukemia (CLL): evidence of in vitro secretion following activation of CLL cells. Leuk Res 34(9): e248-e249.

13. Heinig K, Gätjen M, Grau M, Stache V, Anagnostopoulos I, et al. (2014) Access to follicular dendritic cells is a pivotal step in murine chronic lymphocytic leukemia B-cell activation and proliferation. Cancer Discov 4(12): 1448-1465.

14. Wiestner A, Rosenwald A, Barry TS, Wright G, Davis RE, et al. (2003) ZAP-70 expression identifies a chronic lymphocytic leukemia subtype with unmutated immunoglobulin genes, inferior clinical outcome, and distinct gene expression profile. Blood 101(12): 4944-4951.

15. Pierobon M, Wulfkuhle J, Liotta LA, Petricoin lii EF (2019) Utilization of proteomic technologies for precision oncology applications. Cancer Treat Res 178: 171-187.

16. Hanahan D, Weinberg RA (2011) Hallmarks of cancer: the next generation. Cell 144(5): 646-674.

17. Mayer RL, Schwarzmeier JD, Gerner MC, Bileck A, Mader JC, et al. (2018) Proteomics and metabolomics identify molecular mechanisms of aging potentially predisposing for chronic lymphocytic leukemia. Mol Cell Proteomics 17(2): 290-303. 
18. Thurgood LA, Dwyer ES, Lower KM, Chataway TK, Kuss BJ (2019) Altered expression of metabolic pathways in CLL detected by unlabeled quantitative mass spectrometry analysis. Br J Haematol 185(1): 65- 78.

19. Herishanu Y, Pérez-Galán P, Liu D, Biancotto A, Pittaluga S, et al. (2011) The lymph node microenvironment promotes B-cell receptor signaling, NF-kappaB activation, and tumor proliferation in chronic lymphocytic leukemia. Blood 117(2): 563-574.
20. Vangapandu HV, Chen H, Wierda WG, Keating MJ, Korkut A, et al. (2018) Proteomics profiling identifies induction of caveolin-1 in chronic lymphocytic leukemia cells by bone marrow stromal cells. Leuk Lymphoma 59(6): 1427-1438.

21. Griggio V, Mandili G, Vitale C, Capello M, Macor P, et al. (2017) Humoral immune responses toward tumor-derived antigens in previously untreated patients with chronic lymphocytic leukemia. Oncotarget $8(2)$ : 3274-3288.

For possible submissions Click below: 